\title{
P-0720 TLR4 gene variant as a risk marker of diabetic foot ulcers
}

\author{
*A.M. Raja ${ }^{1}$, M.M. Zafar ${ }^{1}$, M.A. Riaz², J. Zafar ${ }^{3}$, A. Mehmood ${ }^{1}$, M.S. Raja1 , M. Mahmood ${ }^{2}$, G.K. Raja ${ }^{1}$ \\ ${ }^{1}$ University Institute of Biochemistry \& Biotechnology, Pir Mehr Ali Shah Arid Agriculture University Rawalpindi, 46300, Pakistan \\ ${ }^{2}$ Department of Zoology, University of Poonch, Rawalakot, Azad Jammu \& Kashmir, Pakistan \\ ${ }^{3}$ Diabetes Center, Department of Medicine, SZABMU PIMS, Islamabad, Pakistan
}

\begin{abstract}
Background: Diabetes and its secondary complications are the fastest growing chronic diseases worldwide (1). Diabetic foot ulcers are severe forms of diabetic complications resulting in limb amputation, severe health issues or even early death (Fig. 1). The lifetime risks of diabetes especially diabetic foot ulcers (DFU) and imputations are on rapid rise in Pakistan. Though large data exists regarding environmental and genetic risk factors of diabetes, etiology of diabetic foot ulcers genetics are yet to be identified (2). Toll Like Receptor 4 (TLR4) gene encodes a receptor with role in regeneration, immunity and tissue repair. TLR4 gene variants are found associated with infectious and other inflammatory diseases. A TLR4 gene variant, rs4986790, has been reported for impaired wound healing and thus may contribute to diabetic foot ulcers (3).
\end{abstract}

Objective: The present study was designed to identify risk allele of rs4986791 (C/T or Thr399Ile) TLR4 gene SNP and its association with DFU.

Methods: The anthropometric and clinical data were obtained from a total of 260 subjects, including 100 healthy controls, 50 diabetic patients without foot ulcer without Foot Ulcer (DFU-), and 110 Diabetics with Foot Ulcer (DFU+). For microbiota identification in DFU+ cases, pus samples from the infected foot parts were collected and cultured for characterization of frequent microbes. Allele specific For rs498679 SNP based genotyping in all three study groups, Allele Specific Polymerase Chain Reaction (AS-PCR) was performed. The allele and genotype frequencies of rs498679 SNP were calculated and their associations with DFU, anthropometric and clinical risk variables were estimated.

Results \& Discussion: As presented in Table 1, the DFU+ subjects were at an advanced age and had BMI elevated as compared to the than the controls $(<0.0001)$. Based on levels of FBG $(181.8 \pm 66.8)$ and HbA1C $(10.2 \pm 1.73), \mathrm{DFU}+$ subjects also had poor glycemic control as compared to control and DFU- $(\mathrm{p}<0.0001)$ (Table 1$)$. The most frequent microbiota identified in DFU+ subjects was Staphylococcus aureus (26.34\%) showing high sensitivity $(93 \%)$ to Amikacin antibiotic (Table 2 ). The minor $\mathrm{T}$ allele frequency was raised (52\%) in DFU+ compared to controls and DFU- (48\%) groups. Though heterozygous $\mathrm{C} / \mathrm{T}$ genotype seemed common, homozygous $\mathrm{T} / \mathrm{T}$ genotype frequency was higher $(14 \%)$ in DFU+ group (Table 3$)$. The $\mathrm{T}$ allele lacked association with DFUs (Table 4).

Figure 1: Pictures showing foot ulcer and imputation in

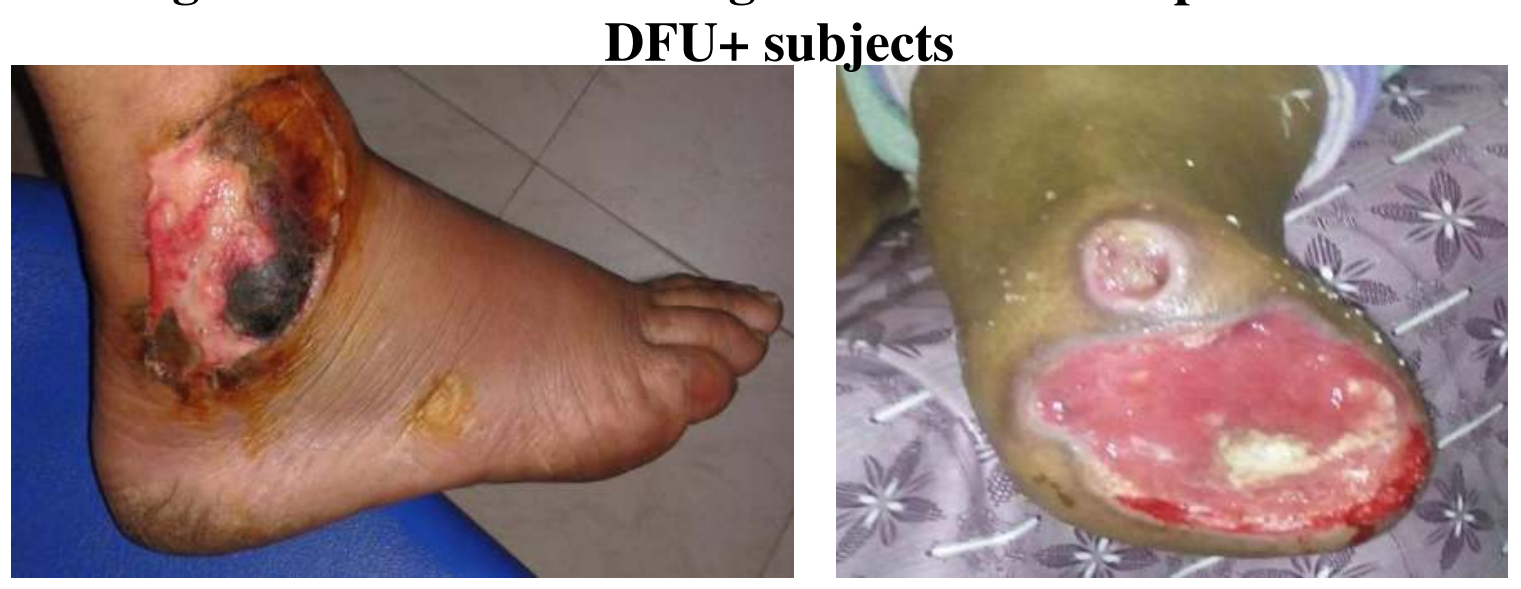

Table 1: Comparison of the anthropometric and clinical variables in study groups

\begin{tabular}{|c|c|c|c|c|}
\hline Variables & Control & DDFU- & DDFU+ & p-Value \\
Age & $46.6 \pm 14.4$ & $52 \pm 12.7$ & $55.3 \pm 10.3$ & $<0.0001$ \\
BMI & $25.7 \pm 3.1$ & $27.3 \pm 3.79$ & $27 \pm 4.86$ & 0.028 \\
FBG & $75.8 \pm 7.6$ & $162.9 \pm 50.1$ & $181.8 \pm 66.8$ & $<0.0001$ \\
HbA1C & $5.57 \pm 0.42$ & $9.11 \pm 1.54$ & $10.2 \pm 1.73$ & $<0.0001$ \\
Creatinine & $1.09 \pm 0.60$ & $1.11 \pm 0.45$ & $1.33 \pm 0.67$ & 0.014 \\
\hline
\end{tabular}

Table 2: Frequency of common microbiota and antibiotic sensitivity in DFU+ subjects

\begin{tabular}{|lc|}
\hline \multicolumn{2}{|c|}{ Microbiota } \\
\hline Microbiota in DFU+ Cases & Frequency (\%) \\
\hline Staphylococcus aureus & 26.34 \\
Pseudomonas aeruginosa & 12.52 \\
\hline Antibiotic Sensitivity (Amikacin) & \\
\hline Staphylococcus aureus & 93 \\
Pseudomonas aeruginosa & 69 \\
\hline
\end{tabular}

Table 3: Allele and genotype frequencies of rs4986791 variant

Table 4: Genotype Association with Study Groups

\begin{tabular}{|cccc|}
\hline \multirow{2}{*}{ Alleles } & \multicolumn{3}{c}{$\begin{array}{c}\text { Frequencies (Proportions) in } \\
\text { Study Groups }\end{array}$} \\
\cline { 2 - 4 } & Control & DFU- & DFU+ \\
\hline C & 0.52 & 0.52 & 0.48 \\
T & 0.48 & 0.48 & 0.52 \\
\hline Genotypes & & & \\
\hline C/C & 0.20 & 0.09 & 0.06 \\
C/T & 0.57 & 0.77 & 0.80 \\
T/T & 0.23 & 0.13 & 0.14 \\
\hline
\end{tabular}

\begin{tabular}{|lcrcccr|}
\hline \multirow{2}{*}{ Genotypes } & \multicolumn{2}{c}{ Control vs DFU- } & \multicolumn{2}{c|}{ Control vs DFU+ } & \multicolumn{3}{c|}{ DFU- vs DFU+ } \\
\cline { 2 - 7 } & OR (95\% CI) & p- valve & OR (95\% CI) & p-valve & OR (95\%CI) & p- valve \\
\hline C/C vs C/T & $3.07(1.07-8.77)$ & 0.052 & $2.37(1.14-4.92)$ & $<0.0001$ & $0.77(0.26-2.28)$ & 0.28 \\
C/C vs T/T & $1.61(0.44-5.88)$ & 0.052 & $0.54(0.18-1.58)$ & $<0.0001$ & $0.33(0.08-1.43)$ & 0.28 \\
C/C vs & $2.69(0.96-7.57)$ & 0.044 & $1.89(0.92-3.87)$ & 0.078 & $0.70(0.24-2.06)$ & 0.5 \\
$\mathbf{C / T}+\mathbf{T} / \mathbf{T}$ & & & & & & \\
\hline
\end{tabular}

\section{Conclusions}

The prominent environmental risk factors identified in Pakistani diabetic foot ulcers were; poor glycemic control, advanced age, male gender, over-weight, serum Creatinine, and a family history of diabetes. The minor T allele frequency of rs4986791 variant of TLR4 gene was raised in DFU patients but it lacked significant association with disease. However in its heretozygous status $(\mathrm{C} / \mathrm{T}), \mathrm{T}$ allele seemed to show significant association with diabetes. Thus our results clearly indicate T allele TLR4 gene as a risk marker of diabetes which could trigger DFU+ under conditions like poor glycemic control.

\section{References:}

1-International Diabetes Federation. Diabetes Atlas, 3rd edn. International Diabetes Federation, Brussels, 2006.

2- Boulton, A.J.M. 2004. The diabetic foot: from art to science. The 18th Camillo Golgi lecture. Diabetic Medicine, 47: $1343-1353$.

3- Kooner J.S., D. Saleheen, X. Sim, J. Sehmi, W. Zhang, P. Frossard, L.F. et al., Chambers. 2013. Genome wide association study in people of South Asian ancestry identifies

six novel susceptibility loci for type 2 diabetes. Europe PMC Funders Group. 43(10): 984-989.

This work was supported by the research grant f20-2255/NRPU/R\&D/HEC funded by the Higher Education Commission of Pakistan
Correspondence: Asad Mehmood Raja

Email: asadscm750@gmail.com

University Institute of Biochemistry and Biotechnology Pir Mehr Ali Shah Arid Agriculture University Rawalpindi, Pakistan 